\title{
Information technology in ophthalmology-experience with an electronic patient record
}

\author{
G W Aylward, D N Parmar
}

The world of medicine has lagged behind many other spheres of life in the exploitation of information technology. Hospitals which may bristle with magnetic resonance imaging scanners and phacoemulsification machines still rely on handwriting to enter clinical information into the notes. The conventional paper medical record, with all its disadvantages, still remains the central depository of medical information for patients in the UK National Health Service, and indeed throughout the world. There have been many applications of information technology (IT) in the NHS over recent years, including several spectacular failures. ${ }^{1}$ Most of these applications have been management driven, and hardly any have been successful in providing a real alternative to the paper record for use by doctors and other groups of clinicians in the secondary care sector. The majority of hospitals in the UK do not even have a computerised patient diagnostic index, which would allow basic questions about case mix to be answered. The primary care sector has fared better, and general practitioners have taken the lead in the clinical use of IT. The majority of general practices have computers in the consultation room, and many paperless practices currently exist. ${ }^{2}$ However, the development of computerised records in the acute hospital sector has been much more difficult, not least because of the increased range and complexity of the information that needs to be recorded.

The pressures for the adoption of an electronic patient record (EPR) in hospitals are now increasing. The process of clinical audit is currently time consuming and inefficient, with missing notes being a major impediment. Audit can be considered to be an algorithmic process. A series of well defined steps is carried out to collate and analyse data in order to answer a specific question. As such, the process is ideally suited for computerisation. However, the data are not generally in a suitable format for a computer. Data collection generally involves analysis of paper records by medical or audit staff, and translation into electronic form. This can be a very inefficient and time consuming process, prone to problems of missing notes, illegible data, and typing inaccuracies. Such inefficiency can compromise the credibility of audit results and may be a barrier to more frequent audit.

The introduction of effective clinical governance is unlikely to succeed without an investment in suitable information technology to support it, particularly when it comes to recording outcomes. ${ }^{3}$ The government white paper "Information for health" has mandated the use of level 3 EPRs in all acute hospitals by $2005 .{ }^{4}$ EPRs have been classified into five groups according to the functions they provide. A level $3 \mathrm{EPR}$ has a fully integrated patient master index, patient administration system and departmental systems, electronic orders for investigations, and electronic prescribing.
This review describes our experience with the design, installation, and use of an EPR in the vitreoretinal service at Moorfields Eye Hospital over the past 18 months. The numerous benefits are described, and the potential for future development is elucidated. In addition, the results of a comparison between a conventional clinical audit and a fully electronic audit are described.

\section{Methods}

A thorough search of available EPR systems failed to discover a commercial product that could satisfy the needs of the department and its vision for an EPR which could provide the functions required. Therefore, a decision was made to develop our own system.

DESIGN

The design philosophy was based on the following principles.

Data ownership

Data should be entered into the computer directly by the clinician. The validity of information is very dependent on the person who enters it. Problems have arisen in the past where third party "coders" have attempted to interpret information written by doctors and convert it into electronic form. ${ }^{5}$ The issue of data "ownership" is very important, and the credibility of data analysis is ultimately dependent on it.

\section{No duplication}

The entry of data onto the computer should be instead of rather than as well as entry into the paper notes. Clinical workload is increasing and there is no extra time available for doctors to duplicate entry of data already written into paper notes. Since paper notes need to exist, at least for the immediate future, a paper entry is required. Therefore, the EPR was designed to print out a paper copy of the electronic entry for filing. This allows continuity of the paper record, particularly for reference by other departments within the hospital, without access to the EPR. The improved flow of information is shown in Figure 1.

No "big bang"

Experience has shown that the sudden replacement of established paper systems with large computer systems is always difficult, and can be disastrous. ${ }^{6}$ The production of a paper copy of each electronic entry allows the introduction of the EPR in an incremental fashion, without disrupting existing paper systems.

FEATURES

The vitreoretinal service at Moorfields Eye Hospital consists of six consultants, 10 junior doctors, a service nurse, and clerical staff. Twenty standard desktop 


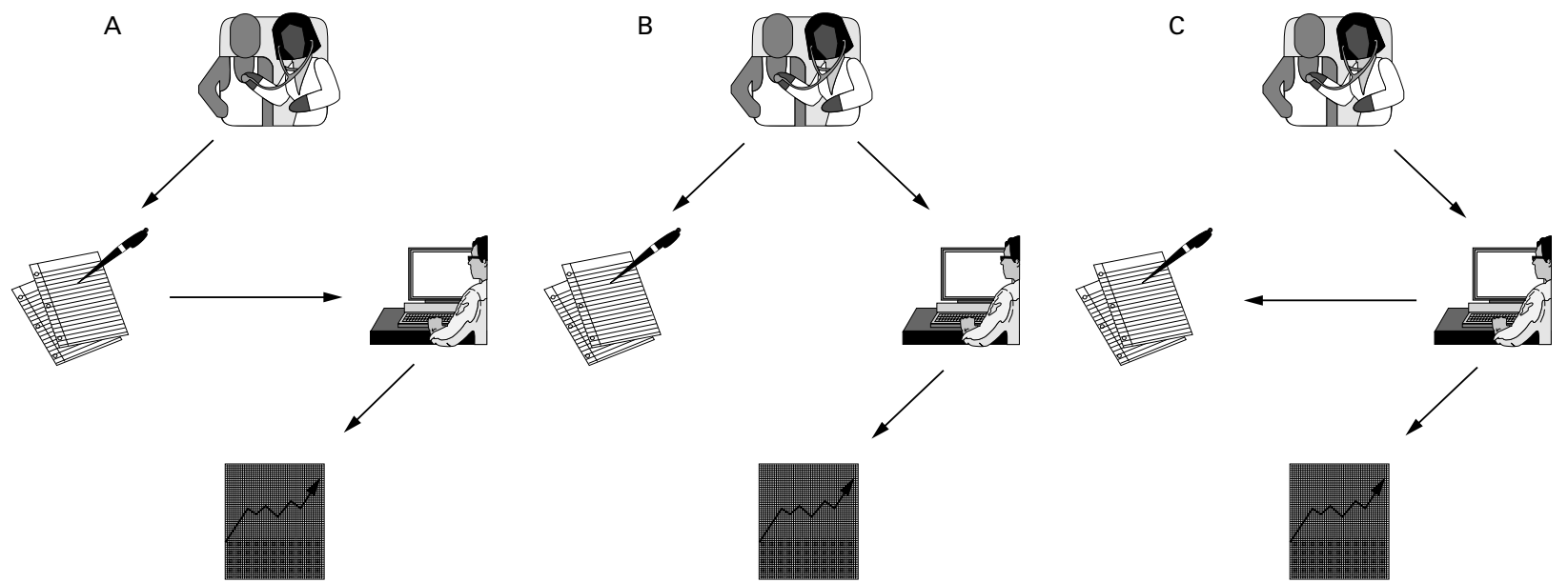

Figure 1 (A) The conventional flow of patient information. Data are recorded in the paper notes, which may then be converted into electronic form for computer analysis, such as audit. (B) Direct data entry by the doctor improves accuracy and data ownership, but must not be at the expense of extra work and data duplication. (C) Arranging for the computer to produce a paper copy achieves the benefits of direct data entry without the disadvantages of duplication.

computers were installed in all the areas where clinical activity takes place, including the outpatient clinic, the ward, and the operating theatre, as well as some consultant and secretarial offices. Figure 2 shows the layout in the outpatient clinic. This arrangement allows direct entry of patient information during or immediately after the consultation with the patient. Each computer is connected to the hospital network so that the data can be stored centrally on a dedicated "server". This allows access from any computer on the network. The technical details of the computer hardware and network are given in Table 1 .

\section{SOFTWARE}

The EPR software was written in Visual FoxPro (Microsoft), a powerful relational database programming language. Wherever possible, standard commercial software programs were linked to it to provide specialised functions. For example, a drawing package to allow entry of diagrams (VISIO), and a standard web browser to allow reading of documents on the NHSnet, or the hospital intranet (Microsoft Internet Explorer). The software allows entry of all the information that is currently written in the paper notes, including diagrams. The data entry is assisted by the computer, according to the context. For example, when the history is being entered, a menu of common items is presented for selection depending on the subspecialty of the doctor using it. For the vitreoretinal service the choice would include floaters, flashing lights, and sudden loss of vision. In addition, the software

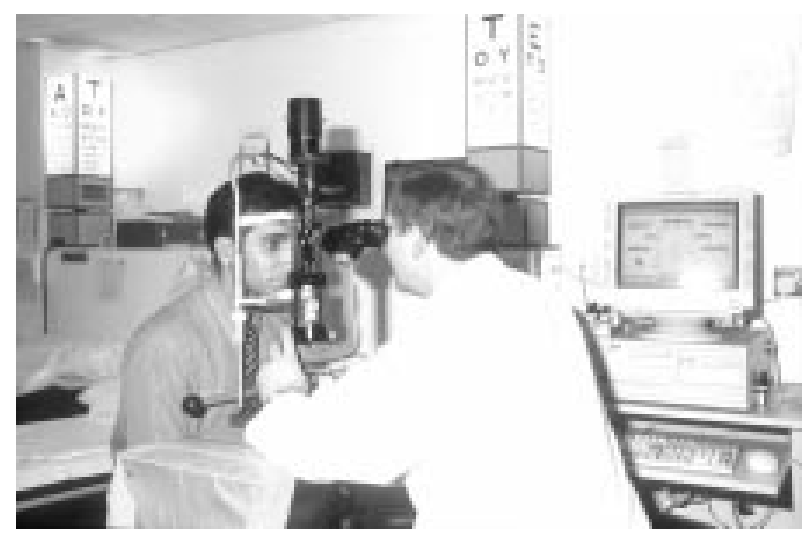

Figure 2 Photograph of the layout of the computer workstation in the outpatient clinic. The keyboard and mouse are arranged so that data can be entered easily at the time of the consultation. provides other functions including a full diagnostic and procedure index using the latest version of the Read codes (version 3), a surgical diary to allow booking of operations, electronic prescribing, and semiautomatic generation of correspondence. Figure 3 shows some screen shots of the system which give an idea of the user interface.

Entry to the software is protected by a user ID and password. In addition, the identity of all users who edit records is recorded. As an additional safeguard, retrospective editing of records is not allowed.

The software began life as a simple diagnostic index, but was developed over a 3 year period, during which time new features were added. After four versions, the software was at a stage where all types of information that are currently written into paper records could be added.

TRAINING

Training consisted of an introductory demonstration to small groups, or individuals, lasting approximately $15 \mathrm{~min}-$ utes. A user ID and password linked to a dummy set of records was specifically provided for training in order to allow users to experiment with the system without fear of damaging existing records. Arrangements were made to ensure the presence of an "expert user" in each clinic so that questions and problems from new users could be quickly addressed.

\section{INTEGRATION INTO WORKFLOW}

The system was introduced in as gentle a manner as possible, since no reduction in the number of patients booked for each outpatient clinic was made. Initially, doctors were encouraged to enter details on new patients only for the first few clinics until they were proficient in the use of the software. The majority of users took two to three clinics to achieve an acceptable level of proficiency.

AUTOMATIC AUDIT

In January 1999 we conducted an audit of primary retinal detachment surgery over a 6 month period. The EPR was

Table 1 Technical details of the computer hardware

\begin{tabular}{ll}
\hline Item & Technical details \\
\hline Client computers & Viglen pentium pro, 200 MHz, 64 mbytes of RAM \\
Operating system & Windows NT 4.0 \\
Database software & Microsoft Visual FoxPro \\
Drawing software & Visio Professional \\
Intranet browser & Microsoft Internet Explorer \\
Network & 10Base T Ethernet \\
Network software & Novell Netware \\
\hline
\end{tabular}



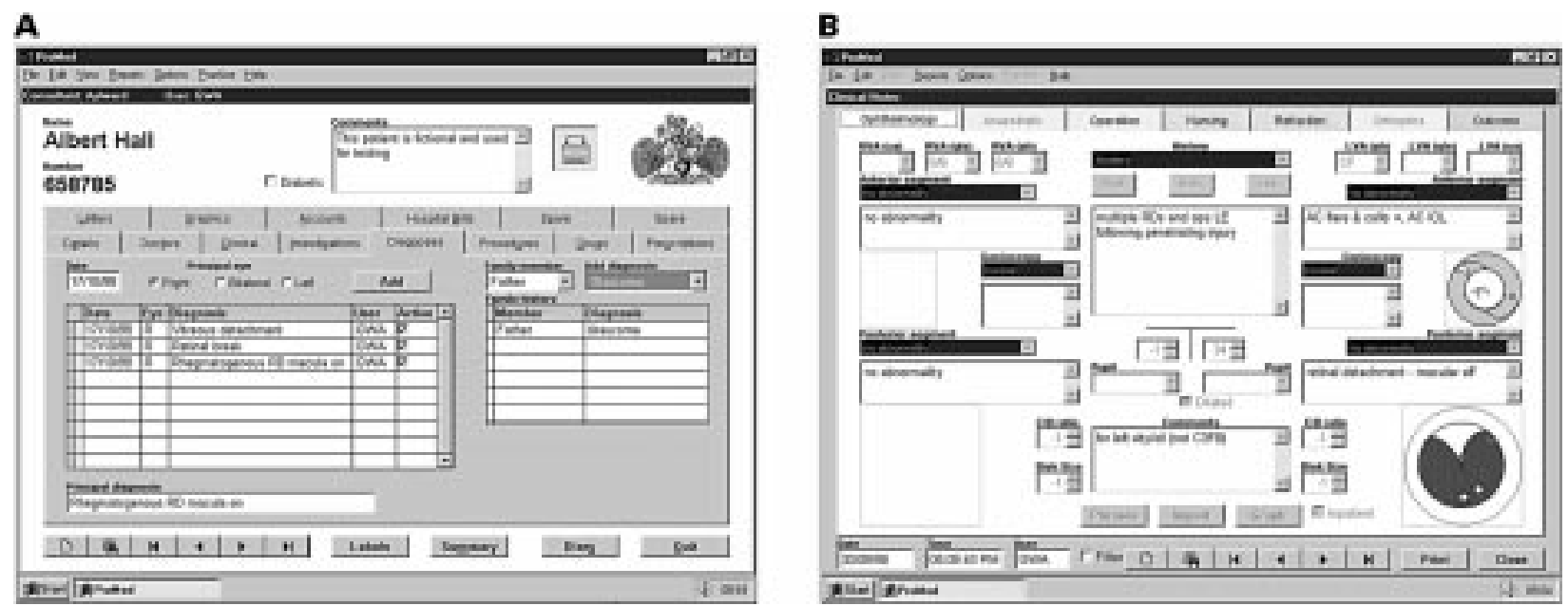

Figure 3 (A) The main screen showing the diagnosis "tab" foremost. A list of Read coded diagnoses is shown. (B) The data entry screen for clinical data, showing dropdown menus with common phrases, and drawings.

used to produce a list of patients with a diagnosis of rhegmatogenous retinal detachment, who had undergone primary surgery at Moorfields over the previous 6 months. The list was then used as the starting point for a standard manual audit, which was undertaken independently by one of the authors (DNP). The time spent on each step of the audit was recorded.

The automatic audit was performed by instructing the EPR to carry out exactly the same steps as the manual audit. A simplified audit protocol was adopted consisting of the data shown in Table 2. This included details on the preoperative state, the findings 3 months postoperatively, details of the type of surgery carried out (vitrectomy or scleral buckle), and the name and grade of surgeon. The EPR then collected the results into a spreadsheet and presented the results graphically. A success was defined as a fully attached retina at 3 months after a single retinal operation.

\section{Results}

USE

The success of the system was assessed by recording its use. The number of patients and the number of letters generated over the initial 10 month period is shown in Figure 4 . The usage climbs rapidly over a 4 month period and

Table 2 Clinical information collected for audit

\begin{tabular}{ll}
\hline Item & Details \\
\hline $\begin{array}{l}\text { Preoperative } \\
\text { Operative }\end{array}$ & $\begin{array}{l}\text { age, sex, eye, visual acuity, macular status } \\
\text { technique (vitrectomy/buckle, etc), surgeon, } \\
\text { consultant } \\
\text { visual acuity, state of retina (attached/detached), } \\
\text { number of retinal operations }\end{array}$ \\
\hline
\end{tabular}

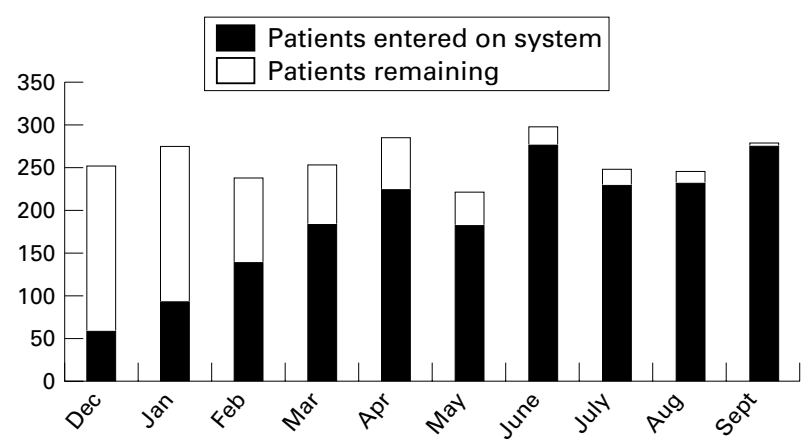

Figure 4 Graph showing the increase in the number of patients entered on the system over the first 10 months. then levels off. This represents saturation of the system, so that all available patients are being entered for the clinics with computer access. The total amount of data entered over the first 12 months, including the number of clinical encounters recorded (corresponding to an entry in the paper notes), is shown in Table 3.

Acceptance of the system by doctors was excellent, with many users making helpful suggestions which were incorporated into the software. Two major problems arose. Firstly, some consultant firms did not have full access to the system, in that some outpatient clinics were conducted in areas without computers. This meant that some clinics were carried out with and some without the EPR. This has now been corrected, but was a major impediment to the adoption of the system. Secondly, the production of clinic letters by the system led to some suspicion from the medical secretariat. This has now largely resolved, with several secretaries using the system instead of their word processor to produce letters.

AUDIT

A total of 65 patients who underwent primary retinal detachment surgery were audited. The time involved in the conventional audit is shown in Table 4 . A total of 14 hours

Table 3 Total number of data entries over a 12 month period

\begin{tabular}{ll}
\hline Item & Number \\
\hline Patients & 2289 \\
Clinic entries & 3751 \\
Procedures & 1908 \\
Letters & 4309
\end{tabular}

Table 4 Time taken to complete elements of a clinical audit, and delay induced

\begin{tabular}{lll}
\hline Task & Time (hours) & Delay (days) \\
\hline Obtain list of patients & 3 & 1 \\
Retrieve notes & $2^{\star}$ & 7 \\
Extract data & 7 & 7 \\
Analyse data & 4 & 4 \\
Total & 16 & 19
\end{tabular}

^Notes retrieval was carried out by audit office staff

Table 5 Details of outcomes which were non-concordant

\begin{tabular}{lll}
\hline Error & Computer & Conventional \\
\hline Failure to count gas injection as second procedure & 1 & 1 \\
Procedure not recorded & 2 & 0 \\
Outcome changed with longer follow up & 2 & 1 \\
Unknown & & 2 \\
\hline
\end{tabular}


of medical time, and 2 hours of clerical time were consumed by the audit. In contrast, the computer program took 45 seconds to process the same data. The results (that is, reattachment rate, final visual acuity, etc) were the same between the two methods, with the exception of eight items where there were differences. These cases were double checked, and the reasons for error are shown in Table 5. It can be seen that there were errors in both audits, but none that would significantly alter the conclusions.

\section{BENEFITS}

The benefits of the system were assessed by discussions with both users and patients. The ability to access the record at any time from any of the workstations was identified as a major benefit. Missing paper notes can be at least frustrating, and at worst dangerous to patient care. The creation of a useful patient index for diagnosis and procedures was also identified as a key benefit.

\section{Discussion}

The results of this study show that an EPR can be introduced into an acute hospital environment successfully, without the trauma and dangers associated with a "big bang". The importance of a graded introduction, with the EPR coexisting with paper records and current hospital forms, cannot be overemphasised, and has been pointed out by other authors. The increase in the use of the system over a 4 month period represents a "group" learning curve, though individual doctors can usually use the system after a month. Training remains an important issue, and sufficient time must be allowed for it, either within or outside the outpatient clinic.

The clear benefits of the system included the ability to have full access to patient information from any workstation at any time. This is a major advantage over conventional paper records and brings with it significant potential savings in human and financial resources. The lack of missing records also makes audit much more valid, since missing paper records can introduce significant bias.

The audit described in this paper was a useful first attempt at a fully automatic audit. Although the number of outcome variables was limited in this case, there is no theoretical limit to the complexity of such audits. The benefits in time saved, and the lack of missing notes will ensure that this type of exercise is developed in the future.

The direct entry of data by doctors is of major importance. The combination of data entry by the clinician and the computerised help given by appropriate software functions has been shown to improve the quality of clinical coding. ${ }^{5}$ It also helps in the avoidance of data duplication, a problem which bedevils paper records.

The choice of Read codes for use in the diagnostic and procedure index has several advantages. It is a rich vocabulary which allows coding of significant detail. It is a "living" coding system which includes mechanisms to rapidly add new codes as they are needed-for example, in the case of new surgical procedures. It is also part of the "language of health" adopted for use in the NHS IM\&T strategy.

The EPR will form the basis of many exciting developments in the further application of information technology to medicine. The quantity of information now available relevant to medicine is increasing at a rate which is beyond the ability of most medical practitioners to absorb. The trend towards evidence based medicine, and the establishment of organisations seeking to produce guidelines, such as the National Institute of Clinical Excellence (NICE), will result in an increased quantity of important information to be read. The use of EPRs will enable an intelligent selection of appropriate information at the right time and in the correct context. For example, the entry of a diagnosis of "posterior vitreous detachment" could trigger the display of the latest trust or national guidelines for its management.

The arrival of the NHSnet will enable faster and more interactive communications with referring general practitioners, and other hospitals. Such communications will be an essential part of the vision of an electronic health record (EHR) described in "Information for Health". The concept is of a brief electronic summary which will enable doctors in one part of the country to discover important background information which might be relevant in the case of emergency treatment, such as allergies, and significant medical history.

In summary, we have shown that an EPR is a practical proposition that can be introduced into an acute hospital relatively easily, and with enormous benefits. It is likely that this sort of EPR will become much more prevalent as we enter the new millennium.

GW AYLWARD D N PARMAR

Moorfields Eye Hospital, City Road, London EC1V 2PD

1 Smith MF, Smart G. Information technology project failures in the NHS. Health Care Computing 1999;1:149-55.

2 Sullivan F, Mitchell E. What difference has general practice computing made to patient care? A systematic review of published reports. BMF 1995; 311:848-52.

3 Eibinder JS, Rury C, Safran C. Outcomes research using the electronic patient record: Beth Israel Hospital's experience with anticoagulation. Computer Applications in Medical Care 1995;819-23.

4 NHS Executive. Information for health. London: DoH, 1998.

4 NHS Executive. Information for health. London: DoH, 1998 . electronic patient record system. Methods of Information in Medicine 1996;35:108-11

6 Atkinson CJ, Peel VJ. Transforming a hospital through growing, not building, an electronic patient record system. Methods of Information in Medicine 1998;37:285-93.

7 Hohnloser JH, Purner F, Kadlec P. Coding medical concepts: a controlled experiment with a computerized coding tool. Medical Informatics 1996;21: 199-206

8 Stuart-Buttle CD, Read JD, Sanderson HF, et al. A language of health in action: Read codes, classifications and groupings. Proceedings of the AMIA Annual Fall Symposium, 1996:75-9. 